Proceedings of Magneto-Optical Recording International Symposium '92, J. Magn. Soc. Jpn., Vol. 17, Supplement No. S1 (1993), pp. 1-6 (1) 1993 by The Magnetics Society of Japan

\title{
EVOLUTION OF MAGNETO-OPTICAL RECORDING MATERIALS
}

\author{
Susumu UCHIYAMA \\ Department of Electronics, Nagoya University \\ 464-01 Nagoya, Japan
}

Abstract - The evolution of magneto-optical recording technology as well as materials is reviewed. A bit density higher than $10^{8}\left[\mathrm{bit} / \mathrm{cm}^{2}\right]$, which is about 3 times higher than that specifled by ISO, has already been realized by employing zcAV technique together with the mark edge recording. The density may attain a value of $10^{\circ}$ [bit/cm $\left.{ }^{2}\right]$ around the beginning of next century by the use of superresolution disk combined with shorter wavelength SHG laser. If it is possible to apply a near field scanning optical microscope method, a recording density of higher than $10^{10}\left[\mathrm{bit} / \mathrm{cm}^{2}\right]$ could be expected. Accompanied by the progress in recording density, a remarkable down sizing for a definite capacity memory or huge capacity image. recorders for video have been realized. On the other hand, as for a task of higher speed required for random access computer memory, the footstep of recent development is not clear and the future is difficult to forecast.

KEYWORDS : MO RECORDING, MATERIAL, DENSITY, SPEED, SIZE

\section{I NTRODUCT I ON}

The magneto-optical (MO) recording devices are now used practically in various fields such as memories for computers, document files, erasable compact disks, etc. In order to widen the field of MO memory application, however, the performances of disk as well as drive must be improved remarkably. As shown in Fig.1, for the use in lap-top computer or word processor, the down sizing may be the key technology. Also as for digital video recorder, a huge capacity is required. Both of these requirements can be realized by the improvement in recording density. The down sizing also contributes to lower price.

In order to compete with the magnetic disk memory, the higher speed is the most important requirement. Since the access speed of MO memory is mostly restricted by stroke time of linear actuator of Mo head. the reduction of stroke length, hence the down sizing, is an effective approach. Apart from the effect of higher density recording, some techniques useful for higher speed devices will also be reviewed briefly.

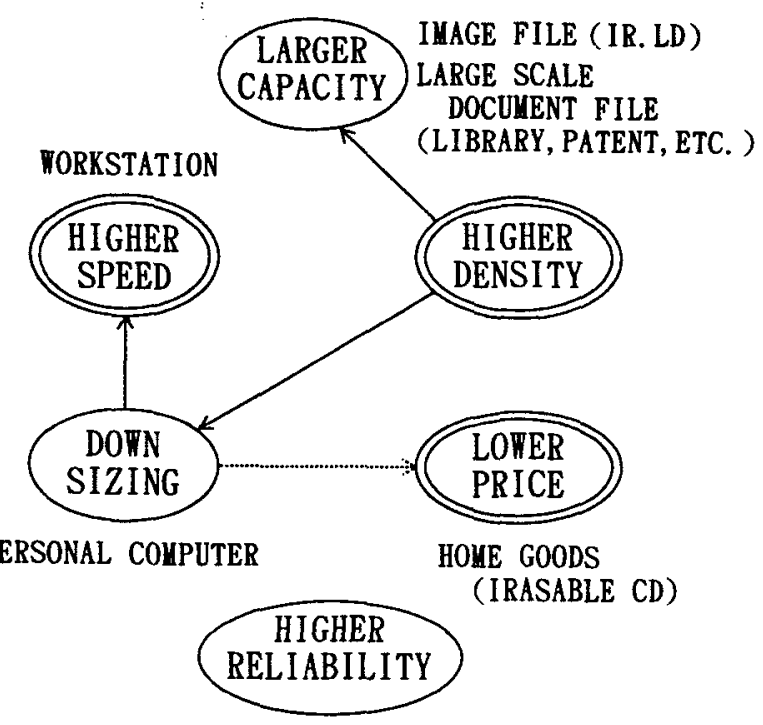

Fig. 1 Requirements for next generation Mo drive

\section{H I GHER RECORD I NG DENS I TY}

\section{1 L initation of Recording Density}

The density of MO recording is limited either by materials or optics. The minimum diameter of a cylindrical domain (a bit) in a perpendicularly magnetized film is given by 


$$
\mathrm{d}_{\mathrm{p}}=\sigma \cdot / \mathrm{M}_{\mathrm{s}} \mathrm{H}_{\mathrm{c}},
$$

where $\sigma$. is the wall energy density, $M_{\text {. }}$ the saturation magnetization, and $H_{c}$ the coercive force. [1] As shown in Fig.2, M. $H_{c}$ product is roughly proportional to the perpendicular anisotropy constant $K_{v}$. Since $\sigma$. is estimated to be about $1 \sim 3 x$ $10^{-3}\left[\mathrm{~J} / \mathrm{m}^{2}\right], \mathrm{d}_{\mathrm{m}}$ is estimated to be smaller than about $0.3[\mu \mathrm{m}]$ for most MO materials such as Tb-FeCo films, Pt/Co multilayers, MnBi films, etc. In this way, $d_{w}$ of these materials is far smaller than the minimum size determined by optical limitation described below, showing that the material is not the limiting factor for recording density at present.

The minimum diameter $\phi$, of the focused laser beam is given by

$$
\phi \ldots i=\lambda / 1.2 \mathrm{NA} \text {. }
$$

where $\lambda$ is the wavelength, NA number of aperture of objective lens. For NA=0.5 and $\lambda=0.78[\mu \mathrm{m}]$ (ISO specification), $\phi$. becomes about $1.3[\mu \mathrm{m}]$. Although a bit with comparable size to $\phi$. is recorded most stably, it is possible to write a bit smaller than $\phi$. by reducing the pulse length of light or magnetic field. In this way, the thermomagnetic writing is also not the limiting factor for the density.

The bit density of present devices is limited mostly from the read out performance. The $S / N$ of read out signal starts to decrease with a bit size given by $d=\phi .$. which corresponds to a density of $\sim 2.4 \mathrm{x}$ $10^{7}\left[\mathrm{bit} / \mathrm{cm}^{2}\right]$ for a track pitch of $1.6[\mu \mathrm{m}]$ (ISO specification).

\section{2 Optical Approach}

The direct method of improving density is to decrease the beam diameter $\phi$. which can be realized either by increasing NA or by decreasing $\lambda$ as known from eq. (2). Actually, NA has been increased from 0.45 for. KDD1 [2], the first MO drive using semiconductor laser, to $0.55 \sim 0.65$ as shown in Tab. 1.

The reduction of $\lambda$ depends on the development in laser technology. It has been changed from 820 [nm] for KDD 1 to 780 [nm] for ISo specification, and will be changed to 532 [nm] of SHG laser within this century. Examples of recent experi-

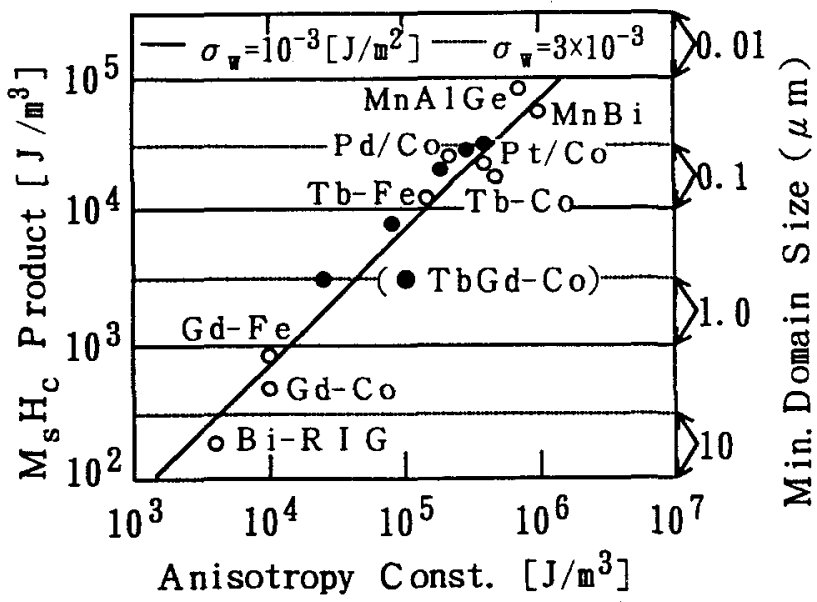

Fig.2 Estimation of minimum domain size in various MO materials in relation with the magnetic anisotropy

Tab. 1 Examples of high density recording experiments

\begin{tabular}{|c|c|c|c|c|c|}
\hline $\begin{array}{c}\lambda \\
{[\mathrm{nm}]}\end{array}$ & NA & $\begin{array}{c}\text { Bit } \\
\text { Length } \\
{[\mu \mathrm{m}]}\end{array}$ & $\begin{array}{c}\text { Track } \\
\text { Pitch } \\
{[\mu \mathrm{m}]}\end{array}$ & Mode & Ref. \\
\hline 780 & 0.50 & 1.00 & 1.6 & LIM & ISO \\
780 & 0.55 & 0.60 & 1.4 & MFM & {$[3]$} \\
780 & 0.53 & 0.55 & 1.6 & MFM & {$[4]$} \\
780 & 0.55 & 0.45 & 1.45 & LIM & {$[5]$} \\
780 & 0.55 & 0.38 & 1.6 & LIM & {$[6]$} \\
687 & 0.55 & 0.5 & 1.2 & LIM & {$[7]$} \\
532 & 0.60 & 0.45 & 1.6 & LIM & {$[8]$} \\
532 & 0.60 & 0.4 & 0.9 & LIM & {$[9]$} \\
458 & 0.60 & 0.38 & 1.6 & LIM & {$[10]$} \\
429 & 0.65 & 0.4 & 0.75 & LIM & {$[11]$} \\
\hline
\end{tabular}

Remarks LIM: Light intensity modulation MFM: Magnetic field modulation

mental results using larger NA and shorter $\lambda$ are summarized in Table.1. The most recent result reported from IBM with $\lambda=$ $429[\mathrm{~nm}]$ and $\mathrm{NA}=0.65[11]$ shows an increase in recording density of 5 times compared with Iso standard drives. (see Tab.1)

Several methods have been developed to read a bit smaller than $d=\phi .$. , which have been called superresolution. The first proposal was to reduce $\phi$. given by Eq. (2) by inserting an aperture plate [12], where the effective diameter of the focused beam was reduced by a factor of 0.8 with a energy loss of $30 \%$.

In conclusion, the increase in density from ISO by means of optics will be as follows. 
$\mathrm{NA}: \quad(0.65 / 0.50)^{2}=1.69$

Superresolution : $1 / 0.80=1.25$

$\lambda=532[\mathrm{~nm}]:(780 / 532)^{2}=2.15$

And, if all of these techniques becomes available, the density will be increased by a factor of 4.5. This number will increase to 7.0 if $\lambda=429$ [nm] is applied.

Recently, another superresolution technique has proposed, where very small domains of the order of 10 [nm] was read by means of scanning optical microscope [13]. In this method, a sharpened optical fiber with a diameter of $\sim 10$ [nm] is used as a scanning optical probe by placing it in closed proximity $(\sim 10 \mathrm{~nm})$ to the surface. The practical application of this technique to Mo drive may require technological innovation, but it is meaningful in a sense that it removes the optical diffraction limitation for read out.

\subsection{P it Edge or Mark Length Recording}

In pit position recording method, which is applied in Iso specification, a bit is stored in a magnetic domain. However, it is possible to store a bit in domaln wall, namely either leading or tailing edges of a mark (magnetic domain) as employed in conventional optical disks. If we apply this pit edge or mark length recording method, the density will be increased by a factor of about 1.5 .

\section{4 S uperresolution $D$ isk}

A novel method of readout beyond the light diffraction limit by means of disk construction was proposed recently [14]. The basic idea is to mask the stored information by magnetic layer (read layer) before reading, and this hidden bit reappears by an appropriate laser illumination. By a proper design of disk, it is possible to read a half size domain compared with conventional disk, namely another factor of 2 can be increased in density.

\section{$2.5 \mathrm{C} \mathrm{L} \mathrm{V}$ or $\mathrm{ZC} \mathrm{A} \mathrm{V} \mathrm{Method}$}

One of the most easiest techniques for increasing density is to make the bit lengths the same from the inner to the outer tracks. In cases of successive read such as audio or video recordings, the method called constant linear velocity (CLV) method is applied rather easily as in minidisk audio recorder appeared quite recently in market [15]. On the other hand, in case of random access memory, the CLV method is difficult to apply. Recently, a method called zoned constant angular velocity (ZCAV) method is proposed, where tracks are divided into several zones from inner to outer and the effec- tive linear velocity is made constant from zone to zone in order to make the bit length equal. By this method, the density increase of up to 1.5 times can be attained.

\section{6 Estimation of Development in Density}

The development in recording density is illustrated in Fig.3, where open circles are data for developmental level drives while closed circles are for commercial level ones. The increase of density in Sony's minidisk drive over ISO is achieved by the employment of $\mathrm{CLV}$ and mark length recording methods. Next generation drive may apply superresolution disk followed by shorter wave length laser.

In all kinds of memory, the density is known to increase almost exponentially year by year. If we apply this rule, the appearance of superresolution disk is estimated to be in 1995, and that of SHG laser around the end of this century as indicated by shaded circles in Fig. 3 .

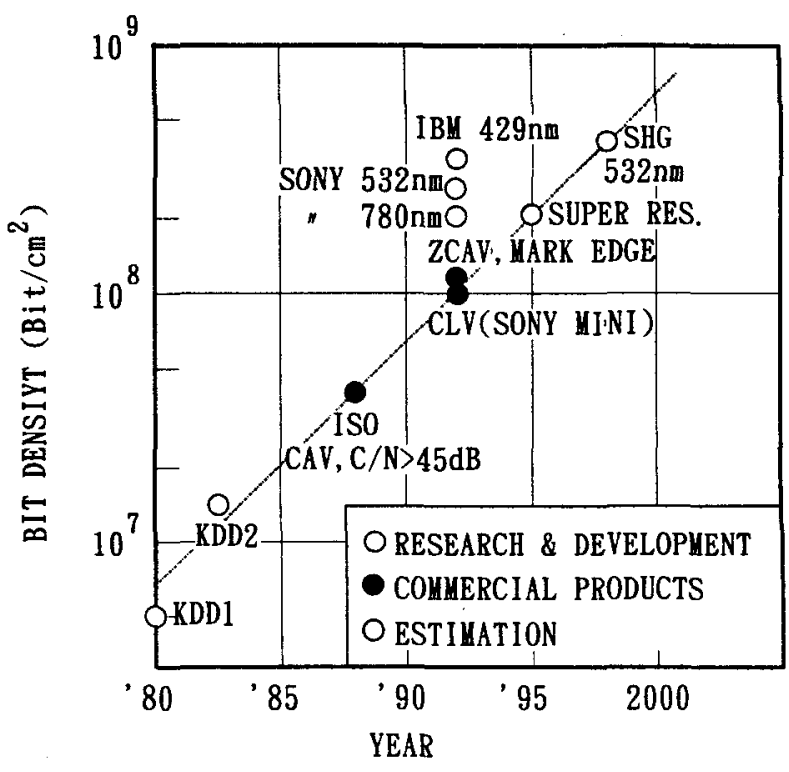

Fig.3 Evolution in recording density 
In Fig.4 is compared the development of recording density in various memories. It is seen that the density as well as its development is similar between MO and contact type magnetic recorder of VTR/DAT. The development in mini hard disk is pretty high, but it will not beyond Mo within this century.

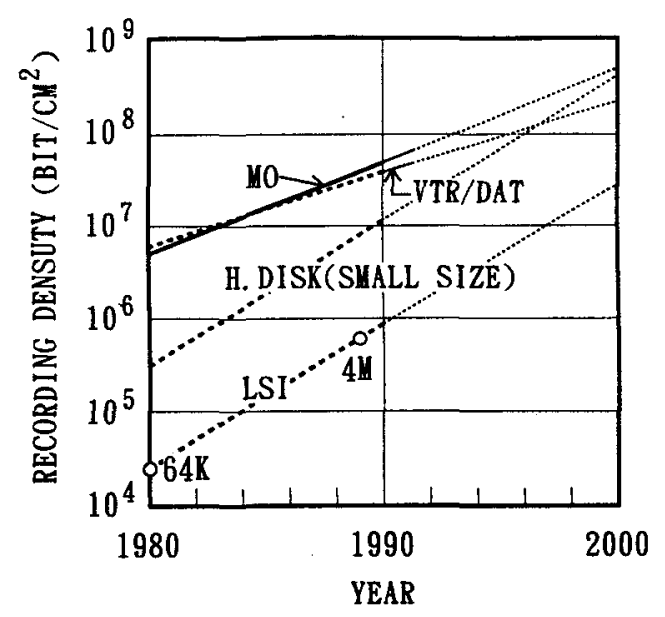

Fig. 4 Progress of bit density in various high density memory

\subsection{Materials for Short Wavelength Recording}

A problem has been pointed out for TbFeCo film that the magneto-optical Rerr rotation $\theta$, decreases with $\lambda$ as shown in Fig.5. This problem seemed to be solved by Pt/Co multilayers [16] [17] [18], since $\theta$. Increases with decreasing $\lambda$ showing a maximum at $\lambda=0.35 \mu \mathrm{m} \mathrm{[19]} \mathrm{[20]} \mathrm{.} \mathrm{A} \mathrm{disk}$ appropriate for $\lambda \sim 0.5$ [nm] was prepared and tested, giving a $\mathrm{C} / \mathrm{N}$ as high as 52 [dB] with a bit length of $0.6[\mu \mathrm{m}]$. A bit as short as $0.3[\mu \mathrm{m}]$ in length was recorded successfully by the magnetic modulation method, and the $c / N$ larger than 42 [dB] was obtained for bit length of 0.4 [ $\mu \mathrm{m}]$ [21].

Another candidate for short $\lambda$ may be amorphous rare earth(RE)-transition metal (TM) alloy films including Nd. In this film, however, the magnetization is too large to become perpendicular to the film plane. By using multilayer structure of NaGd /FeCo, the perpendicularly magnetized film can be prepared with larger $\theta$, compared with Tb-FeCo as seen in Fig.5 [22].

The most classic Mo material, MnBi, is found to have $\theta$, almost 5 times as large as that of $\mathrm{Tb}-\mathrm{FeCo}$ at 380 [nm] as shown in Fig.5 [23]. Problems for this film are in coarse crystalites giving rise to large medium noise and in phase change between room to Curie temperatures, etc. These faults seem to be reduced by the doping of Al, Si etc. [23] [24] and film preparation method [25]. Recently, MnB1 MO disk is prepared again without any doping and is tested, giving a $\mathrm{C} / \mathrm{N}$ of $32 \mathrm{~dB}$ [26] .

As for MO recording materials, garnets may be a strong rival of RE-TM. Unfortunately, the lack of the data on magnetic anisotropy and wall energy density makes difficult to estimate limiting density.

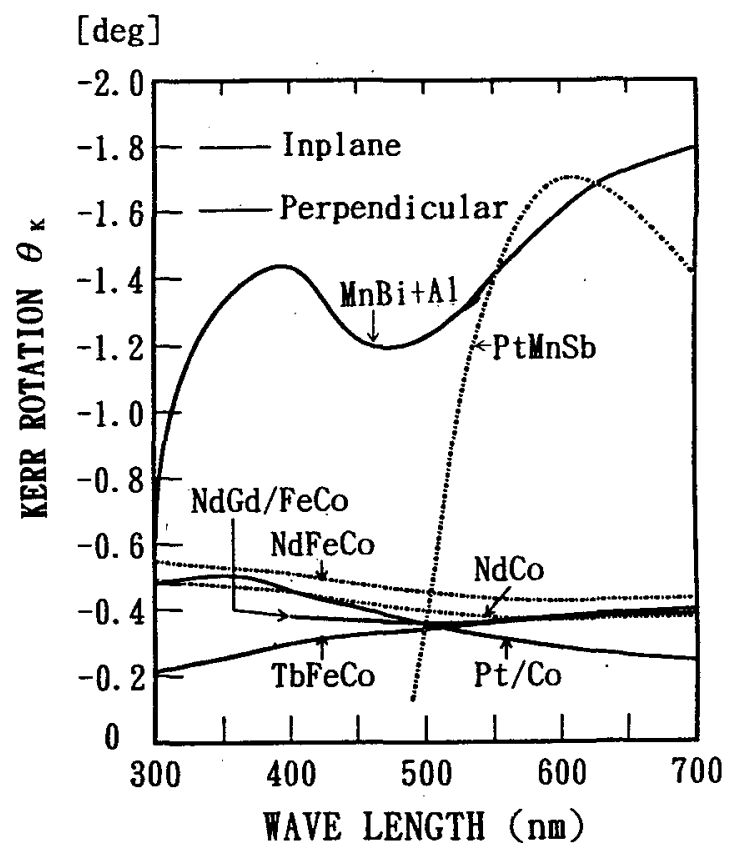

Fig.5 Rerr spectra for various magnetooptical materials

\section{H IGHER SPEED}

To increase the share of $M O$ in the field of computer memory, the speed must be improved first of all. Efforts have continued for all possible techniques in mechanical, optical, disk structure etc. From the material side, however, the only contribution is in the overwritable disks. proposals so far reported are summarized in Table 2.

Since the speed limiting factor in random access memory is in the mechanical movement of optical head in radial direction, the efforts on materials cannot improve the speed markedly. In this connection, the development of smaller sized, 
Proceedings of Magneto-Optical Recording International Symposium '92, J. Magn. Soc. Jpn., Vol. 17, Supplement No. S1 (1993), pp. 1-6 (1) 1993 by The Magnetics Society of Japan

light weight optical head is the key technology to increase the actuator apeed in radial direction.

on the other hand, the use of smaller size disk as a result of increased desity will results in higher speed mostly due to the decrease in stroke length. The down sizing is also effective in increasing rotational speed of disk, which has been improved from 1000 [rpm] for $20[\mathrm{~mm} \phi] \mathrm{disk}$ to $3000[\mathrm{rpm}]$ for $130[\mathrm{~mm} \phi] \mathrm{disk}$ and will be $5400[\mathrm{rpm}]$ for $64[\mathrm{~mm} \phi]$ disk. In this way, the mean seek time of 90 35 [ms] for Iso standard drives for 130 [mm $]$ disk is decreased to 22 [ms] for 90 [mm] disk [45] or 20 [ms] for minidisk of 64 [mm] [46].

Parallel readout using multibeam laser array [44] can increase the data transfer rate required in real time digital image recording and reproducing by the same factor as the number of array.

Different from the case of density, however, it is difficult to forecast the future development in speed at this stage. Therefore, only the recent progress in mean seek time is summarized in Fig. 6 .

Tab.2 Various overwrite techniques

\begin{tabular}{|c|c|c|}
\hline \multicolumn{2}{|c|}{ Classification } & References \\
\hline \multirow{2}{*}{$\begin{array}{l}\text { Magnetic } \\
\text { Field } \\
\text { Modulation }\end{array}$} & DC Light Beam & $\begin{array}{lll}{[27]} & {[28]} & {[29]} \\
{[30]} & {[31]}\end{array}$ \\
\hline & Pulse L. Beam & [32] [33] [34] \\
\hline \multirow{5}{*}{$\begin{array}{c}\text { Light } \\
\text { Intensity } \\
\text { Modulation }\end{array}$} & Single Layer & $\begin{array}{llll}{[35]} & {[36]} & {[37]} \\
{[38]} & & \end{array}$ \\
\hline & Double Layer & [39] \\
\hline & Triple Layer & [40] \\
\hline & Quadri Layer & [41] \\
\hline & Leakage Flux & [42] \\
\hline Multibeam & Pseudo Method & [43] [44] \\
\hline
\end{tabular}

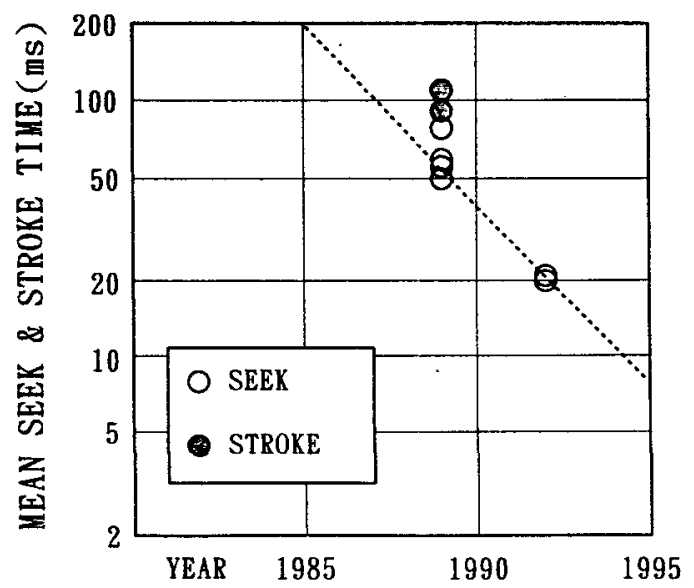

Fig.6 Evolution for higher speed
4. DOWN SIZING AND LARGER CAPACITY

With the development of recording density, the down sizing in drives has been achieved as shown in Fig.7, where the capacity is kept almost constant. For this progress, also the down sizing efforts in both optics and mechanics have been playing very important roles.

For image storage use, the capacity can be increased with the same rate as that in density if the drive size the same. It is expected to produce a 20 [mm $\operatorname{misk}$ which can record the digital video image of 2 hours in near future.

For special use such as library, patent file etc., the capacity can be increased as you wish by increasing the numbers of disks per spindle and drives, and/or by using jukebox type drive. However, the theme may be beyond of this scope.

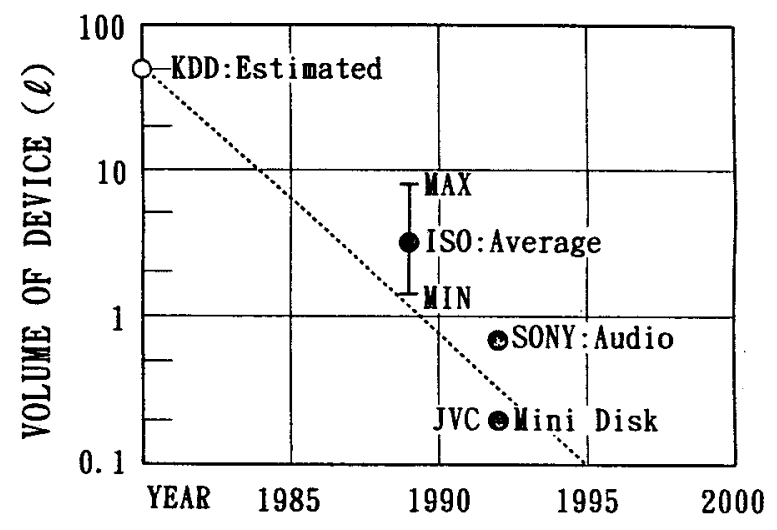

Fig.7 Tendency of down sizing

\section{S UMMARY}

To the end of higher recording density, an improvement of factor of 3 has already been realized compared with iso standard drives, and another factor of $2 \sim 4$ will be realized within this century. Here, the limiting factor is not in recording media but mostly in semiconductor lasers.

For speed, materials are also not a major limiting factor. The improvement depends mostly on mechanical and optical efforts. However, the down sizing as the result of increasing recording density contributes markedly in increasing speed. 
A large number of efforts for overwritable disk will also contribute to the higher speed.

For home goods, the first concern is in disk price, which mostly depends on materials including substrate and on the disk processing technique. Here, again the down sizing may give an effective mean. For video use, a higher density as well as a higher data transfer rate is the primary concerns. The latter may be solved easily by the multibeam technique.

In conclusion, the recent progress in MO recording in relation with higher density is so remarkable that the future is very promising and expecting.

\section{R E F E R E N C E S}

[1] G.B.Huth: IBM Res.\& Devlopm., 18, 1974, p. 100

[2] N.Imamura and C.Ohta: Jpn.J.Appl. Phys. 19, 1980, p.L731

[3] H.Sukeda et al.: Tech.Report IEICE, 1989, MR 89-56

[4] M.Hiramatsu et al.: Jpn.J.Appl. Phys.,15, Part 1, 2B, 1992, p.535

[5] M.Tobita et al.: Digest SPIE, ODS, San Jose, USA, 1992, P.82

[6] S.Ohnuki et al.: Digest Opt.Memory Symp., Yokohama, Jpn., 1992, p.119

[7] S.Sugaya et al.: Digest Opt.Memory Symp., Yokohama,Jpn., 1992, p.59

[8] M.Takahashi et al.: Digest SPIE, ODS, San Jose,USA, 1992, p.123

[9] Y.Sabi et al.: Digest of $16 \mathrm{th}$ Conf. Magn.Soc.Jpn., Nagoya, 1992, p.434

[10] W.B.Zeper et al.:Dig.of Intermag 1992, St.Louis, USA, DA-03

[11] A.G.Dewey et al.: Post Deadline Paper of Digest SPIE., ODS, San Jose, 1992

[12] Y.Yamanaka et al.: Jpn.J.Appl. Phys.,28,Suppl.28-3,1989, p. 197

[13] R.E.Betzig et al.: Digests 37th MMM Conf., Houston, USA., 1992, CBO 1

[14] K.Aratani et al.: Proc.sPIE, 1449, ODS Topical Meeting, 1991, P.209

[15] H.Yoshimura et al., Digest MORIS'92. 1992, Tucson, USA, p.99

[16] P.F.Carcia et al.: Appl.Phys.Lett., 47, 1985, p. 178

[17] W.B.Zeper et al.: IEEE Trans.Magn., MAG-25, 1989, P.3764

[18] Y.Ochiai et al.: IEEE Trans.Magn., MAG-25, 1989, P. 3755

[19] K.Nakamura et al.: J.Magn.Soc.Jpn., 13,1989, p.319

[20] S.Hashimoto et al.: Jpn.J.Appl. Phys..28, 1989, p.L1824

[21] Y.Sabi et al.:Digest 16th Ann.Conf. Mag.Soc.Jpn., Nagoya, 1992, p.434

[22] X.Y.Yu et al.: Digest MORIS' 92 , 1992, Tucson, USA, p.62
[23] G.Q.Di et al.: J.Magn.Soc.Jpn., 15 1992, P. 191

[24] Y.J.Wang et al.: J.de Phys., C8, 1988, p. 1725

[25] M.Masuda et al.: Jpn.J.Appl.Phys., 26,1987, P.707

[26] M.Nakada et al.: Dig. 16th Ann.Conf. Mag.Soc.Jpn., Nagoya, 1992, p.409

[27] F.Tanaka et al: : Tech.Report of JIEE, MAG-86-95, 1986, p.53

[28] N.Ohta et al.: Tech.Report IEICE, CPM87-89, 1987, p.31

[29] R.Ando et al.: Tech.Report IEICE, MR87-37, 1987, p.13

[30] Y.Ohtani et al.: Tech.Report IEICE, MR88-49, 1988, p.1

[31] K.Yamada et al.: Proc.SPIE, 899, 1988 , p. 160

[32] D.Rugar : IEEE Trans.Magn., MAG-24, 1988, p.666

[33] Watanabe et al.: Proc. Opt. Memory Symp. $88,1988, p .47$

[34] Kataoka et al., Proc. Opt. Memory Symp.' 88,1988, p.53

[35] H.P.Shieh et al.: Appl.Phys.Lett., 49, 1986, p.473

[36] P.Hansen: Appl.Phys.Lett., 50, 1987, p.356

[37] D.Lugar et al.: Appl.Phys.Lett. , 52,1988, P. 1537

[38] C.M.Savage et al.: Appl.Phys.Lett., $52,1988, \mathrm{P} .1277$

[39] J.Saito et al.: Jpn.J.Appl.Phys.. 26, Suppl.26-4, 1987, p. 155

[40] R.Aratani et al.: Proc.SPIE, 1078, 1989 , p. 258

[41] T.Fukami et al.: J.Appl.Phys., 67, 1989, p.4415

[42] H.A.M.Berg et al.: IEEE Trans.Magn.. 25,1989, p. 4036

[43] M.Hartman et al.: IEEE Trans.Magn.. MAG-20, 1984, P. 1016

[44] K.Trazawa et al.. J.Magn.Soc.Jpn.. $11,1987, \mathrm{p} .217$

[45] Y.Murakami et al.: Digest of MORIS '92, Tucson, USA, 1992, p.100

[46] Y.Haneji : Digests Opt. Mem. Symp., Yokohama, Jpn., 1992, P.13 